\title{
Toward Personalized Cell Therapies by Using Stem Cells 2012
}

\author{
Ken-ichi Isobe, ${ }^{1}$ Herman S. Cheung, ${ }^{2}$ and Ji $\mathrm{Wu}^{3,4}$ \\ ${ }^{1}$ Department of Immunology, Nagoya University Graduate School of Medicine, 65 Tsurumai-cho, Showa-ku, Nagoya 466-8550, Japan \\ ${ }^{2}$ Biomedical Engineering Department, College of Engineering, University of Miami, Coral Gables, FL, USA \\ ${ }^{3}$ Key Laboratory for the Genetics of Developmental and Neuropsychiatric Disorders (Ministry of Education), Bio-X Institutes, Shanghai \\ Jiao Tong University, Shanghai 200240, China \\ ${ }^{4}$ School of Life Sciences and Biotechnology, Shanghai Jiao Tong University, Shanghai, China
}

Correspondence should be addressed to Ken-ichi Isobe, kisobe@med.nagoya-u.ac.jp

Received 12 November 2012; Accepted 12 November 2012

Copyright (C) 2012 Ken-ichi Isobe et al. This is an open access article distributed under the Creative Commons Attribution License, which permits unrestricted use, distribution, and reproduction in any medium, provided the original work is properly cited.

The iPSCs technology opens the possibility of personalized cell therapies for treating human disease and/or repairing the damaged tissues. Multipotent adult stem/progenitor cells including mesenchymal stem cells (MSCs) also have the potential to use personalized cell therapy.

One target of stem cell therapy is to apply genetic disorders, which are difficult to treat by other medical ways. Q. Jiang et al. showed interesting experiment by using MSC. They transfected Abcc6 gene into MSC and differentiated to hepatic cells and transplanted Pseudoxanthoma Elasticum model mouse.

Myocardial infarction and ensuing heart failure are the leading causes of mortality in western countries. "Cardiospheres" are self-assembling spherical clusters of cells obtained by outgrowth from cardiac explants in the primary ex vivo tissue culture. CSs placed in a new culture dish disassembled and gave rise to a monolayer of CS-derived cells (CDCs) that formed second-generation CSs. CDCs could be expanded as monolayers on fibronectin. L. Barile et al. introduced the first clinical trial of autologous c-kit+/LinCSCs for treatment of heart failure which improved left ventricular function of ischemic heart disease.

One of the most important applications of stem cell therapy is end-stage renal disease (ESRD). More than 290,000 ESRD patients are currently undergoing dialysis in Japan. S. Yokota et al. reviewed recent works, which target the stem cell therapies to ESRD.

K. Chenard et al. have summarized current clinical therapy of craniofacial reconstruction by BMPs; they proposed future personalized therapy by culturing MSCs with BMPs.

In this second edition, we found the paper, which discuss or examine the controversial issues for achieving cell therapy safely and effectively. P. Nascimento reviewed the problems to be analyzed before practical use of stem cells: methods for stem cells culture, teratogenic or tumorigenic potential, cellular dose, proliferation, senescence, karyotyping, and immunosuppressive activity. Recently, it has been shown that MSCs have immunoregulatory functions. E. Ivanova-Todorova et al. reported that MSCs lead to increased expression of FoxP3 regulatory $\mathrm{T}$ cells.

\section{Acknowledgments}

We thank all of the authors for submitting their papers in this special issue and appreciate their efforts and time in processing their papers. We also thank all the reviewers for carefully checking the submitted papers.

Ken-ichi Isobe Herman S. Cheung $\mathrm{Ji} W u$ 

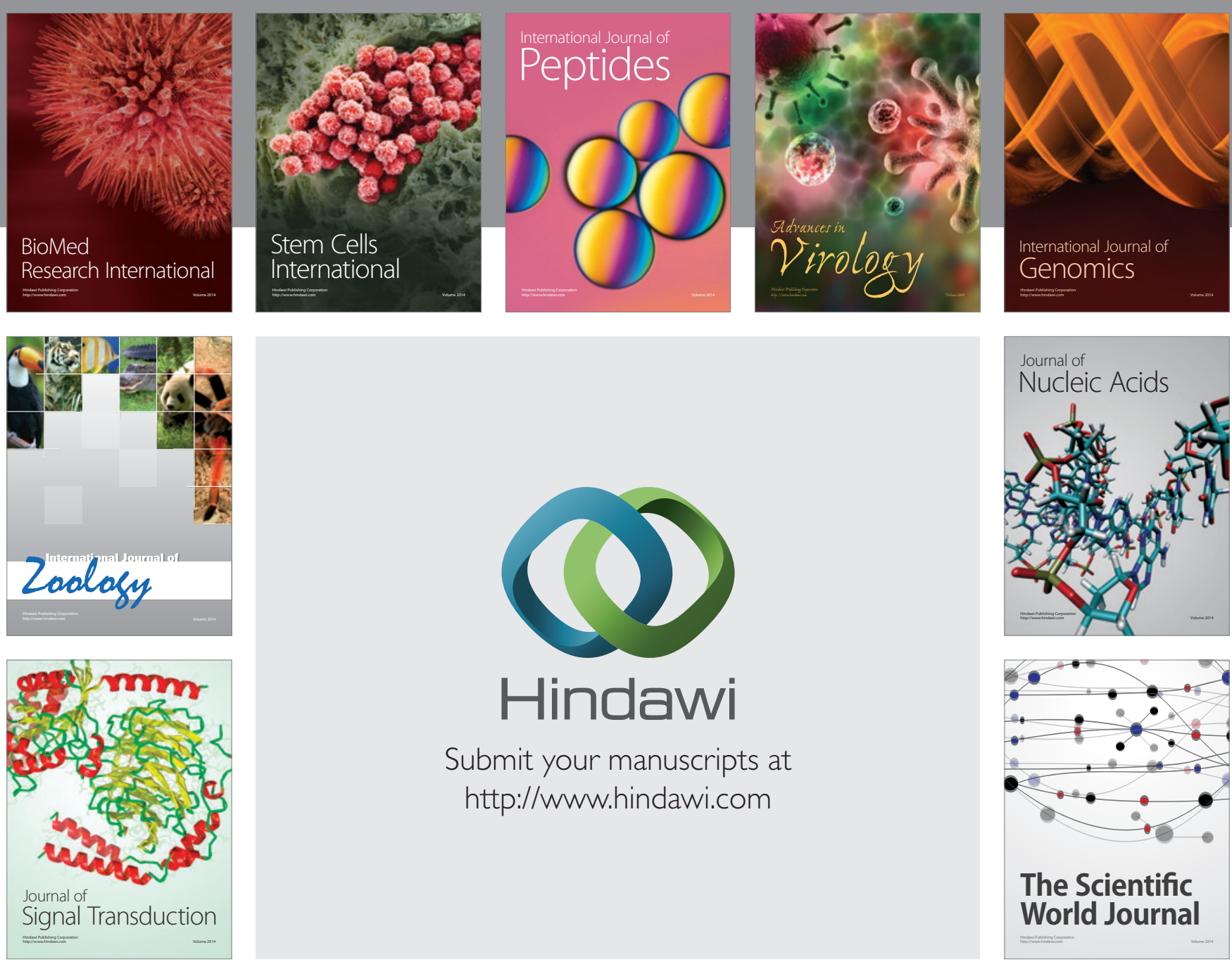

Submit your manuscripts at

http://www.hindawi.com
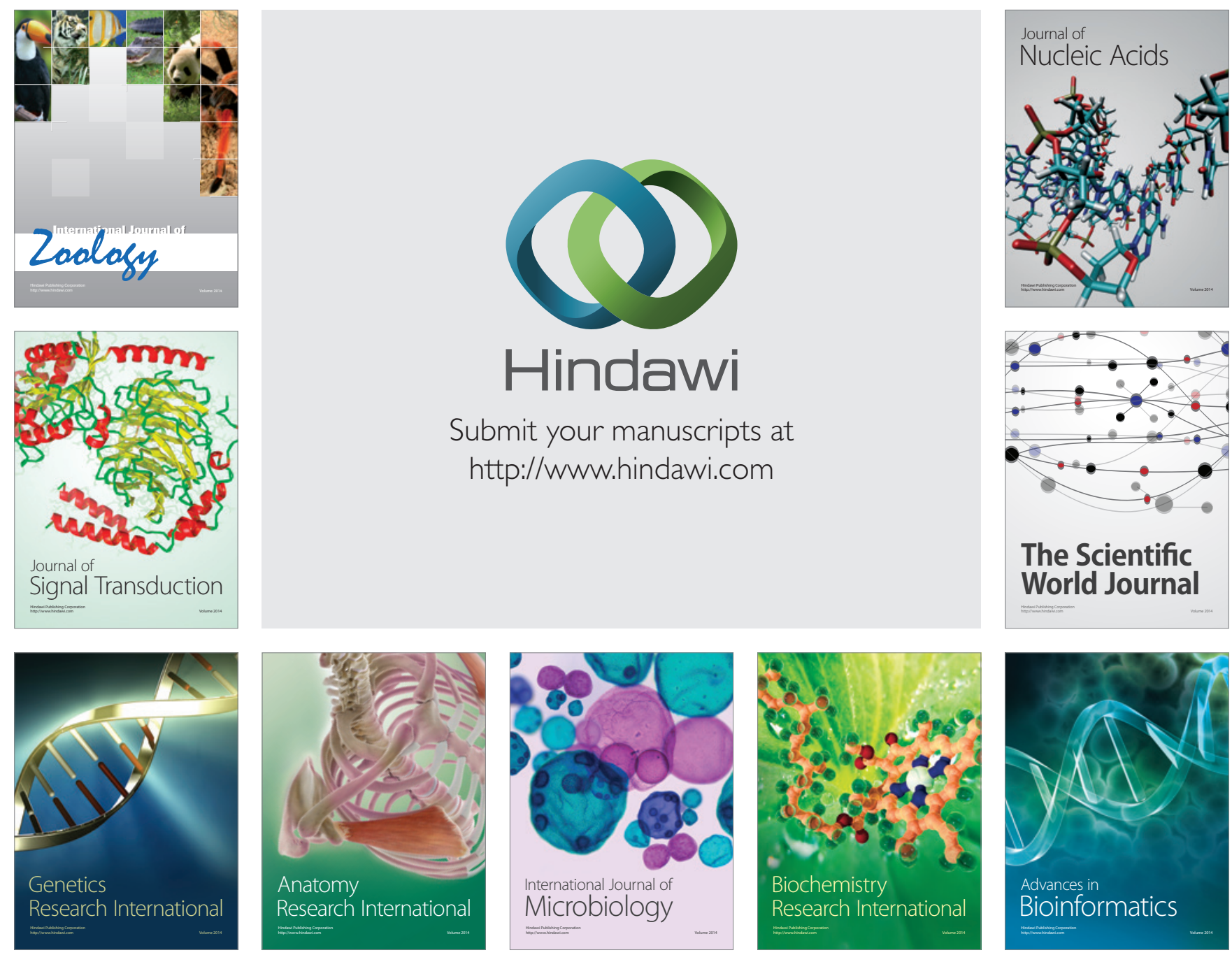

The Scientific World Journal
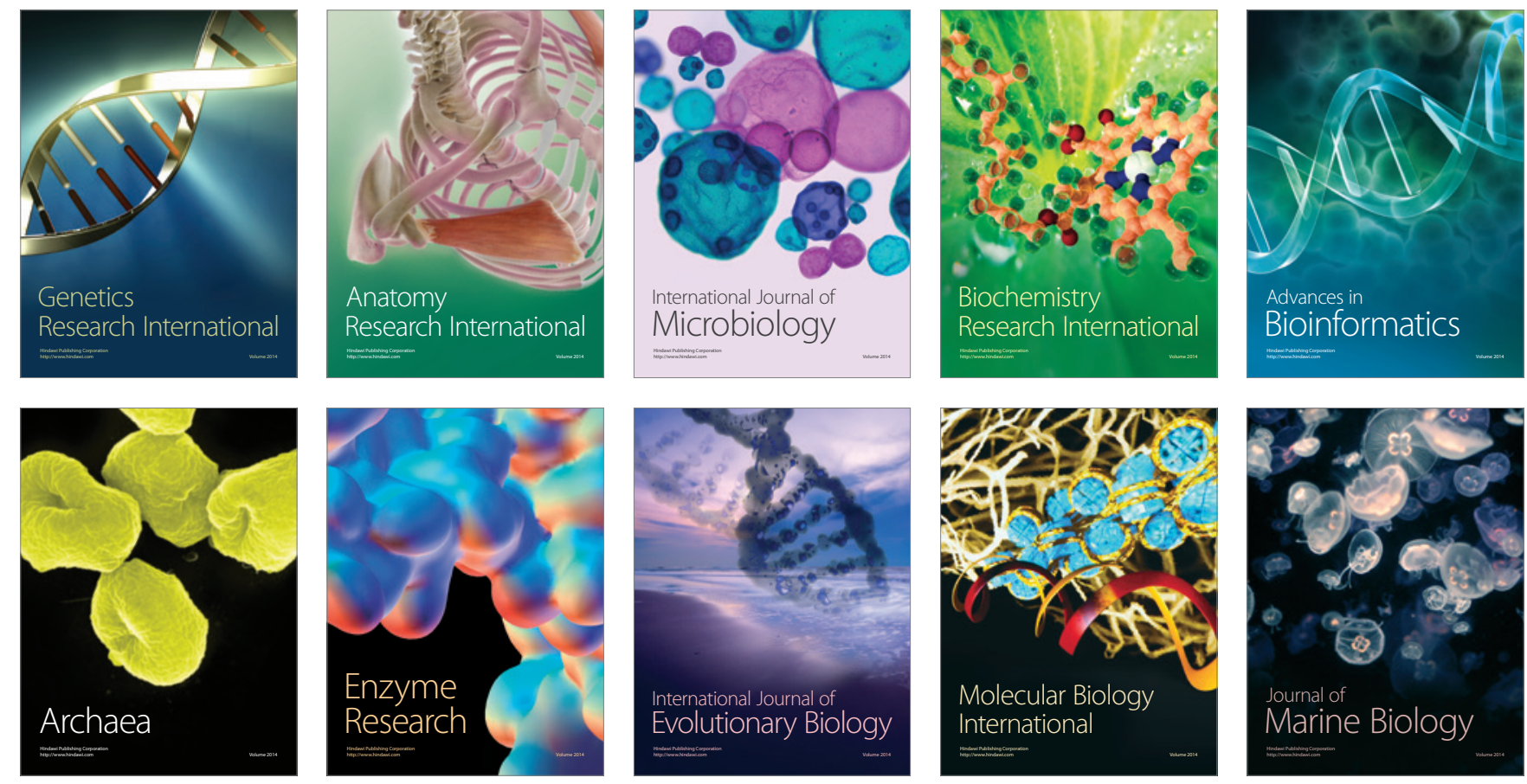